\title{
First-Principles Investigation of the Effect of M-Doped (M = Zr, Hf) TiCoSb Half-Heusler Thermoelectric Material
}

\author{
Gaili Sun ${ }^{1,2}$, Yuanyuan Li1 ${ }^{1}$ Xinxin Zhao², Yiming Mi1*, Lili Wang2* \\ ${ }^{1}$ College of Chemistry and Chemical Engineering, Shanghai University of Engineering Science, Shanghai, China \\ ${ }^{2}$ College of Fundamental Studies, Shanghai University of Engineering Science, Shanghai, China \\ Email: "yimingmi@sues.edu.cn, "llwang@sues.edu.cn
}

Received 27 November 2015; accepted 26 December 2015; published 29 December 2015

Copyright (C) 2015 by authors and Scientific Research Publishing Inc.

This work is licensed under the Creative Commons Attribution International License (CC BY). http://creativecommons.org/licenses/by/4.0/

(c) () Open Access

\begin{abstract}
The M-doping ( $M=\mathrm{Zr}$, $\mathrm{Hf}$ ) effects on the electronic structures and thermoelectric performance of TiCoSb were studied by first-principles calculations. The band structure analysis shows that substituting Ti with $M$ does not change the band structures of these systems significantly. Most of the M-doped systems have a lower band gap value than that of $\mathrm{TiCoSb}$; especially $\mathrm{Ti}_{0.5} \mathrm{Zr}_{0.5} \mathrm{CoSb}_{\text {has }}$ the lowest energy band gap value of $0.971 \mathrm{eV}$. Besides, the amplitudes of the density of states in the region of the valence bands for $M$-doped systems show a similar but slightly higher value than TiCoSb. Those suggest that these compounds could have better thermoelectric performance than TiCoSb. The phonon dispersion relations show that the larger mass of $\mathrm{Zr} / \mathrm{Hf}$ with respect to $\mathrm{Ti}$ lowers the optical modes and induces mixing with the acoustic branches. Our calculations offer a valuable insight on how to characterize complicated crystal structures of thermoelectric materials and optimize the material composition.
\end{abstract}

\section{Keywords}

Half-Heusler Compound, Thermoelectric, First-Principles, Electronic Structure

\section{Introduction}

The potential candidates of ternary half-Heusler $(\mathrm{HH})$ compounds for high performance thermoelectric materials have attracted great attentions in recent years. Thermoelectric materials have played an important role in the solution to today's energy crisis, which can directly convert waste heat into usable electricity in an environmentally-friendly manner [1]. The efficiency of thermoelectric materials at temperature $\mathrm{T}$ is given by the dimen-

${ }^{*}$ Corresponding authors.

How to cite this paper: Sun, G.L., Li, Y.Y., Zhao, X.X., Mi, Y.M. and Wang, L.L. (2015) First-Principles Investigation of the Effect of M-Doped ( $\mathrm{M}=\mathrm{Zr}$, Hf) TiCoSb Half-Heusler Thermoelectric Material. Journal of Materials Science and Chemical Engineering, 3, 78-86. http://dx.doi.org/10.4236/msce.2015.312012 
sionless figure of merit ZT $=S^{2} \sigma \mathrm{T} / \kappa$, where $S$ is the Seebeck coefficient, $\sigma$ is the electrical conductivity, $\kappa$ is the thermal conductivity, which consists of the electron thermal conductivity $\left(\kappa_{e}\right)$ and the lattice thermal conductivity $\left(\kappa_{l}\right)$ [1]-[5]. A better thermoelectric performance means a higher ZT values; therefore, the requirement of a high $\mathrm{ZT}$ value is to maximize the power factor $\left(\mathrm{PF}=S^{2} \sigma\right)$ and minimize the thermal conductivity [2].

TiCoSb-based half-Heusler compound with relatively large Seebeck coefficient and electrical conductivity has been investigated extensively for a decade. It was revealed experimentally that the maximum power factor is $23 \mu \mathrm{Wcm}^{-1} \cdot \mathrm{K}^{-2}$ at $850 \mathrm{~K}$ for TiCoSb-based alloys [3]. However, one prominent problem of this compound for thermoelectric application is its relatively high thermal conductivity $\left(\kappa \approx 10 \mathrm{Wm}^{-1} \cdot \mathrm{K}^{-1}\right)$ [4]-[7]. For this reason, many efforts have been made to reduce its lattice thermal conductivity. It was reported that substitution with isoelectronic elements in the Ti-site lattice can cause additional phonon scattering, and thus have a good balance between a high PF and an effectively reduced $\kappa$ [7]-[11]. Hohl et al. found that the mass disorder in the M-site lattice of compounds causes additional phonon scattering, thereby reducing the thermal conductivity to $6 \mathrm{Wm}^{-1} \cdot \mathrm{K}^{-1}$ at room temperature for $\mathrm{Zr}_{0.5} \mathrm{Hf}_{0.5} \mathrm{NiSn}$ [11]. Xie et al. decreased the lattice thermal conductivity by doping MCoSb-based compounds with $\mathrm{Ni}$, and a maximum $\mathrm{ZT}$ value of 0.51 was obtained for $\mathrm{Ti}_{0.5} \mathrm{Zr}_{0.25} \mathrm{Hf}_{0.25} \mathrm{Co}_{0.95} \mathrm{Ni}_{0.05} \mathrm{Sb}$ at $813 \mathrm{~K}$ [12]. Additionally, the reduction of thermal conductivity can be achieved by using nanoparticle phonon scattering centers [13]-[16], introducing point defects [17] [18] and reducing the grain size [19].

However, the exact mechanism of substitution with isoelectronic elements in the Ti-site lattice in different doping concentrations of TiCoSb is not completely understood. Therefore, it is necessary to understand the influence of the M-doped ( $\mathrm{M}=\mathrm{Zr}$, Hf) TiCoSb and to evaluate the electronic structures and thermoelectric performance of TiCoSb under different $\mathrm{M}$ doping concentrations. In this work, the first-principles calculations were performed to study the electronic structure of M-doped TiCoSb under different doping concentrations. By using the result of the electronic structure calculation, thermoelectric properties of TiCoSb are analysed based on the density functional theory.

\section{Computational Details}

First-principles calculations of TiCoSb-based half-Heusler compounds were performed within the density functional theory (DFT) framework as implemented invia Vienna Ab initio Simulation Package (VASP) code [20]-[22]. The projector augmented wave (PAW) method were used to span out the valence electron density, using the generalized gradient approximation (GGA) in the scheme of Perdew-Burkr-Ernzerhof (PBE) was adopted for the exchange-correlation functional [23]. For the plane wave basis set a cutoff energy of $400 \mathrm{eV}$ and an energy convergence criterion of $10^{-6} \mathrm{eV}$ for self-consistency was used throughout. The Brillouin-zone was sampled $21 \times 21 \times 21$ Monkhorst-Pack k-point mesh for the primitive cells of Ti/Zr/HfCoSb. For the unit cell of M-doped TiCoSb ( $\mathrm{M}=\mathrm{Zr}$, Hf), we have used $4 \times 4 \times 4$ Monkhorst-Pack k-point mesh. The phonon dispersion relations for undoped and doped TiCoSb are obtained from the Phonopy package.

\section{Results and Discussions}

\subsection{Crystal Structures}

The half-Heusler XYZ phases are ternary compounds with the MgAgAs structure. These compounds crystallize in the face-centered cubic (fcc) structure with the space group $F \overline{4} 3 m$ (No. 216). The unit cell of TiCoSb is shown in Figure 1(a). It can be seen, the unit cell contains four formula units with the Co, Sb and Ti atoms located at the 4a: $(0,0,0), 4 \mathrm{~d}:(3 / 4,3 / 4,3 / 4)$ and $4 \mathrm{c}:(1 / 4,1 / 4,1 / 4)$ positions, respectively [24]. To simulate the $\mathrm{M}$ ( $\mathrm{M}=\mathrm{Zr}$, Hf) doped on TiCoSb system, we studied the substitution of Ti by $\mathrm{M}$ in different doping concentrations. Therefore, we chose three doping concentrations of $x=25 \%, 50 \%$ or $75 \%$ in $\mathrm{Ti}_{1-x} \mathrm{M}_{x} \mathrm{CoSb}$, which means substituting 1, 2 or 3 out of $4 \mathrm{Ti}$ atom in TiCoSb unit cell. The primitive cell of $\mathrm{Ti}_{0.5} \mathrm{M}_{0.5} \mathrm{CoSb}$ contains six atom and has a tetragonal structure [1] with space group $P \overline{4} m 2$, as shown in Figure 1(d). Moreover, ZrCoSb and HfCoSb correspond to the doping concentrations of $x=100 \%$ in $\mathrm{Ti}_{1-x} \mathrm{M}_{x} \mathrm{CoSb}$, which crystal structures are the same as that of TiCoSb.

The ground state lattice constant was calculated with relaxation of both atomic positions and lattice parameters. The relaxed lattice constants of undoped and doped TiCoSb are summarized in Table 1. For Ti/Zr/HfCoSb, 


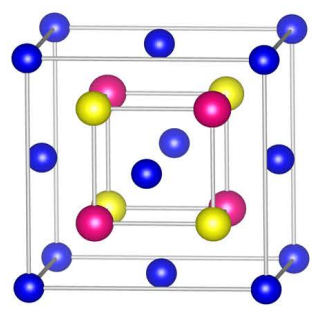

(a)

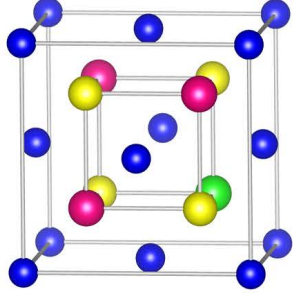

(b)

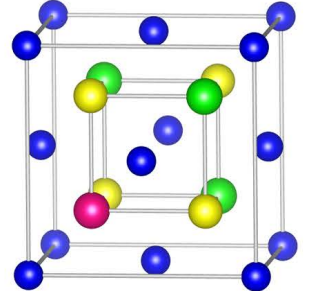

(c)

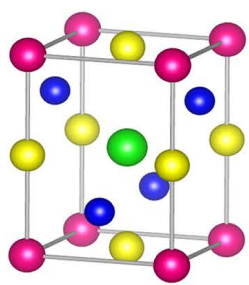

(d)

Figure 1. The crystal structure of TiCoSb based half-Heusler compound: (a) TiCoSb; (b) $\mathrm{Ti}_{0.75} \mathrm{M}_{0.25} \mathrm{CoSb}$; (c) $\mathrm{Ti}_{0.25} \mathrm{M}_{0.75} \mathrm{CoSb}$; (d) $\mathrm{Ti}_{0.5} \mathrm{M}_{0.5} \mathrm{CoSb}$. Blue spheres denote Co atoms, yellow spheres denote Sb atoms, purple spheres denote Ti atoms and green spheres denote the dopant $\mathrm{M}(\mathrm{M}=\mathrm{Zr}, \mathrm{Hf})$.

Table 1. Lattice constants $(a)$, formation enthalpies $\left(\Delta H_{\text {form }}\right)$ and energy band gaps $\left(\mathrm{E}_{\mathfrak{g}}\right)$ of undoped and $\mathrm{M}$ doped TiCoSb half-Heusler compounds.

\begin{tabular}{cccccccc}
\hline Compounds & $a(\AA)$ & $\Delta H_{\text {form }}(\mathrm{eV})$ & $\mathrm{E}_{\mathrm{g}}(\mathrm{eV})$ & & $a(\AA)$ & $\Delta H_{\text {form }}(\mathrm{eV})$ & $\mathrm{E}_{\mathrm{g}}(\mathrm{eV})$ \\
\hline $\mathrm{TiCoSb}$ & 5.89 & -- & 1.050 & & & & \\
$\mathrm{Ti}_{0.75} \mathrm{Zr}_{0.25} \mathrm{CoSb}$ & 5.94 & -0.67 & 0.993 & $\mathrm{Ti}_{0.75} \mathrm{Hf}_{0.25} \mathrm{CoSb}$ & 5.93 & -0.67 & 1.031 \\
$\mathrm{Ti}_{0.5} \mathrm{Zr}_{0.5} \mathrm{CoSb}$ & 6.00 & -0.70 & 0.971 & $\mathrm{Ti}_{0.5} \mathrm{Hf}_{0.5} \mathrm{CoSb}$ & 5.98 & -0.68 & 1.028 \\
$\mathrm{Ti}_{0.25} \mathrm{Zr}_{0.75} \mathrm{CoSb}$ & 6.05 & -0.73 & 0.981 & $\mathrm{Ti}_{0.25} \mathrm{Hf}_{0.75} \mathrm{CoSb}$ & 6.02 & -0.70 & 1.056 \\
$\mathrm{ZrCoSb}^{6}$ & 6.10 & -- & 1.061 & $\mathrm{HfCoSb}$ & 6.06 & -- & 1.135 \\
\hline
\end{tabular}

the optimized lattice constants are in good agreement with the experimental [25] and other theoretical results [24] [26], with slight overestimates typical of the GGA [27]. The above results indicate that the crystal model was rational and could be used for computational prediction. For $\mathrm{Ti}_{0.5} \mathrm{M}_{0.5} \mathrm{CoSb}, a$ is equivalent to the $c$ axis of its tetragonal cell. As seen from this table, with the increasing of $\mathrm{M}$ doping concentration, the calculated lattice constants of doped systems increase gradually.

Commonly, the structural stability of crystal can be evaluated by its formation enthalpy $\Delta H_{\text {form. }}$. A negative formation enthalpy indicates that the crystal can exist stably. In order to investigate the stability of M-doped TiCoSb system, the formation enthalpies $\Delta H_{\text {form }}$ are calculated by using Equation (1) [28]:

$$
\Delta H_{\text {form }}=1 / 3\left[E_{\text {tot }}\left(\mathrm{Ti}_{1-x} \mathrm{M}_{x} \mathrm{CoSb}\right)-(1-x) \mathrm{E}_{\text {solid }}(\mathrm{Ti})-x E_{\text {solid }}(\mathrm{M})-E_{\text {solid }}(\mathrm{Co})-E_{\text {solid }}(\mathrm{Sb})\right]
$$

where $E_{\text {tot }}\left(\mathrm{Ti}_{1-x} \mathrm{M}_{x} \mathrm{CoSb}\right)$ refers to the total energies of doped systems. $E_{\text {solid }}$ represents the total energies per atom in the bulk structure. The calculated formation enthalpy $\left(\Delta H_{\text {form }}\right)$ of doped compounds are shown in Table 1. It can be seen that the calculated formation enthalpies of these compounds are all negative, which indicates they can exist stably. According to the thermodynamic theory, the doped TiCoSb systems of M substituted different concentration can exist stably.

\subsection{Effects of M-Dopant on Electron Transport Properties}

We performed the energy band structures and density of states calculations for undoped and doped TiCoSb half-Heusler compounds, as a first step towards a microscopic understanding of their electronic structures. Figure 2 shows the calculated energy band structures for Ti/Zr/HfCoSb half-Heusler compounds. The band structure is plotted with 150 uniform $\boldsymbol{k}$ points along certain high symmetry directions in the irreducible Brillouin zone (IBZ). It can be seen that all three compound exhibit narrow-gap semiconductors. TiCoSb and HfCoSb have indirect gaps between $\Gamma$ point and $\mathrm{X}$ point from Figure 2(a) and Figure 2(c), while ZrCoSb has indirect gaps between $L$ point and $X$ point as shown in Figure 2(b). The calculated $E_{g}$ values of these three compounds are shown in Table 1. As we see, all three compounds are narrow band gap semiconductors. The largest $\mathrm{E}_{\mathrm{g}}$ value is $1.135 \mathrm{eV}$ for HfCoSb, while TiCoSb gives the smallest $\mathrm{E}_{\mathrm{g}}$ value of $1.050 \mathrm{eV}$. These numbers are in good agreement with those found previously [24] [26].

In Figure 3, we show the calculated energy band structures for Zr-doped TiCoSb half-Heusler compounds. The band structure is plotted with 66 uniform $\boldsymbol{k}$ points at the utmost along certain high symmetry directions in 


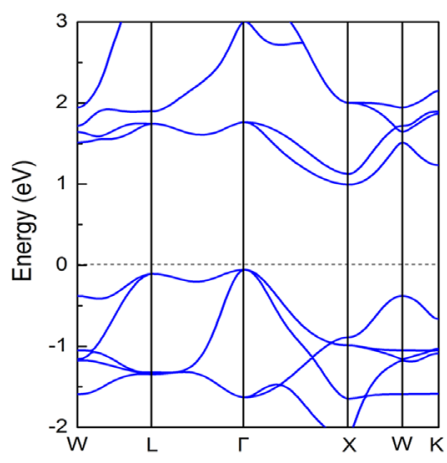

(a)

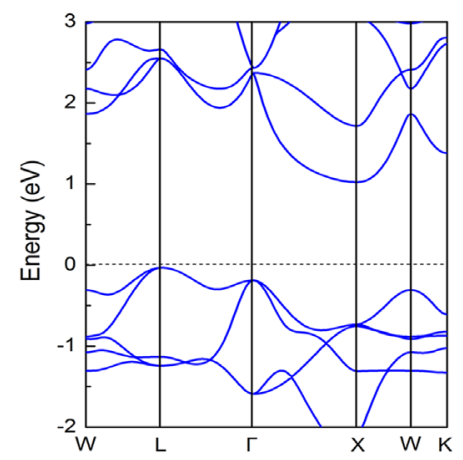

(b)

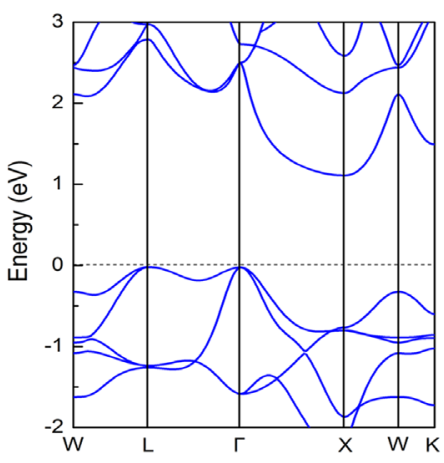

(c)

Figure 2. The band structures of half-Heusler compounds: (a) TiCoSb; (b) ZrCoSb; (c) HfCoSb.

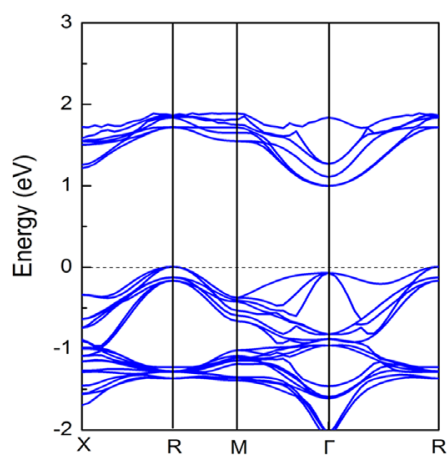

(a)

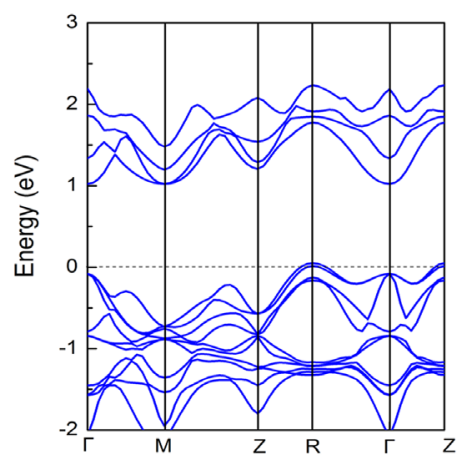

(b)

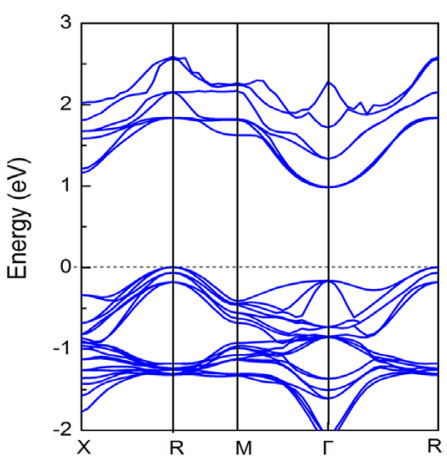

(c)

Figure 3. The band structures of $\mathrm{Zr}$-doped TiCoSb half-Heusler compounds: (a) $\mathrm{Ti}_{0.75} \mathrm{Zr}_{0.25} \mathrm{CoSb}$; (b) $\mathrm{Ti}_{0.5} \mathrm{Zr}_{0.5} \mathrm{CoSb}_{\text {; }}$ (c) $\mathrm{Ti}_{0.25} \mathrm{Zr}_{0.75} \mathrm{CoSb}$.

IBZ. It can be seen that isoelectronic doping TiCoSb system does not change its band structure. All three doped systems exhibit narrow-gap semiconductors, and the indirect gaps between $\mathrm{R}$ point and $\Gamma$ point. The calculated $\mathrm{E}_{\mathrm{g}}$ values of these three compounds are shown in Table 1. With the increasing of the Zr doping concentration, the energy gap value of these compounds increased firstly and then decreased, and $\mathrm{Ti}_{0.5} \mathrm{Zr}_{0.5} \mathrm{CoSb}$ has the lowest $\mathrm{E}_{\mathrm{g}}$ value of $0.971 \mathrm{eV}$. As discussed by Singh, the magnitude of thermopower can decrease at high temperatures and low doping due to the compensation between holes and electrons when the band gap is small [29]. Therefore, the relatively small gap of $\mathrm{Ti}_{0.5} \mathrm{Zr}_{0.5} \mathrm{CoSb}$ suggests that this compound could have better thermoelectric performance than TiCoSb.

In Figure 4, we show the calculated energy band structures for Hf-doped TiCoSb half-Heusler compounds. Like Zr-doped system, the band structure is plotted with 66 uniform $\boldsymbol{k}$ points at the utmost. It can be seen that all three compounds exhibit narrow-gap semiconductors. For both compounds, the valence band (VB) maximum appears at the $\mathrm{R}$ point and conduction band $(\mathrm{CB})$ minimum at the $\Gamma$ point. The calculated $\mathrm{E}_{\mathrm{g}}$ values of these three compounds are also shown in Table 1. Similarly, with the increasing of the Hf doping concentration, the energy gap value of these compounds are increased firstly and then decreased. $\mathrm{Ti}_{0.5} \mathrm{Hf}_{0.5} \mathrm{CoSb}$ has the lowest $\mathrm{E}_{\mathrm{g}}$ value of $1.028 \mathrm{eV}$, but this value is larger than that of $\mathrm{Ti}_{0.5} \mathrm{Zr}_{0.5} \mathrm{CoSb}$. In the $\mathrm{M}$ doping region of $25 \%$ to $75 \%$, most of the doping systems have lower band gap values compare with TiCoSb system, especially $\mathrm{Ti}_{0.5} \mathrm{Zr}_{0.5} \mathrm{CoSb}$ has the lowest value. The relatively small band gap values suggest that these compounds might have better thermoelectric performance than TiCoSb.

Figure 5 shows the density of states (DOS) of the undoped and Zr-doped TiCoSb half-Heusler compounds. In Figure 5, we just show the DOS for these five compounds near Fermi energy $\left(E_{F}\right)$ level, because the thermoelectric properties are mainly associated with the electronic states near $E_{\mathrm{F}}$. Obviously, the number of atoms in unit cells of different compounds is different, to get comparable results, a normalized processing of the calculated DOS (as shown in Figure 5(a)) is used to obtain the DOS of these five compounds under the same number of atoms (as shown in Figure 5(b)). As can be seen from Figure 5(b), the DOS of these half-Heusler com- 


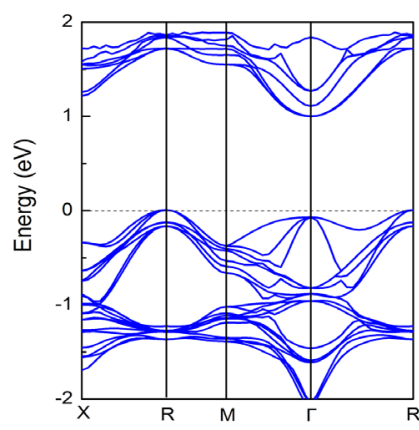

(a)

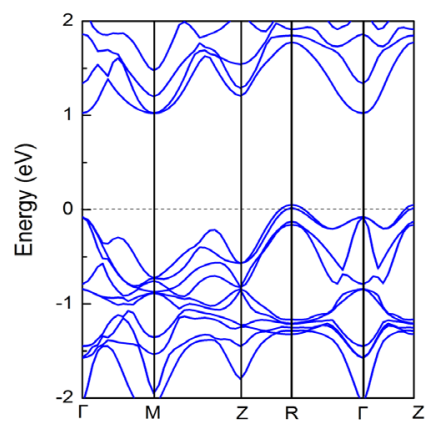

(b)

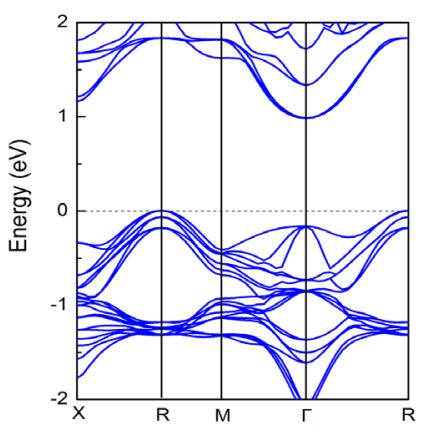

(c)

Figure 4. The band structures of Hf-doped TiCoSb half-Heusler compounds: (a) $\mathrm{Ti}_{0.75} \mathrm{Hf}_{0.25} \mathrm{CoSb}$; (b) $\mathrm{Ti}_{0.5} \mathrm{Hf}_{0.5} \mathrm{CoSb}$; (c) $\mathrm{Ti}_{0.25} \mathrm{Hf}_{0.75} \mathrm{CoSb}$.

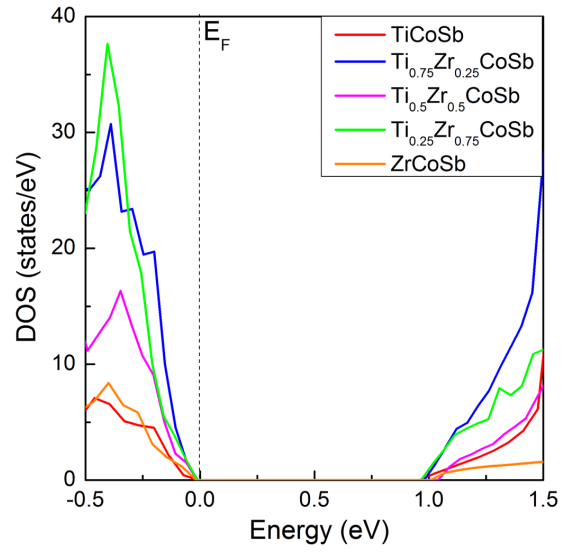

(a)

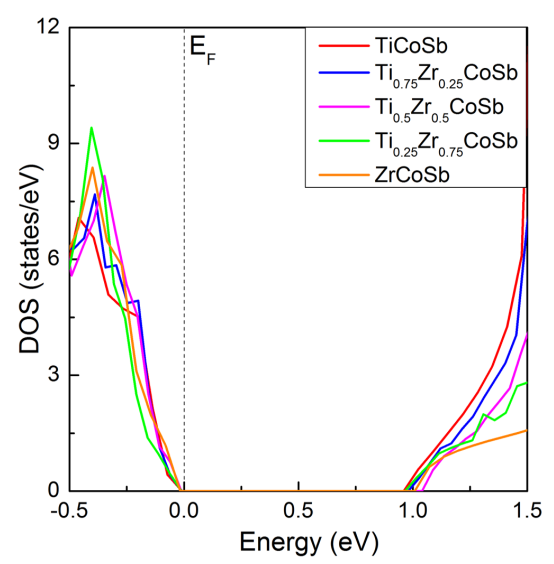

(b)

Figure 5. The density of states of undoped and Zr-doped TiCoSb without normalized (a) and with normalized (b).

pounds both exhibit sharp peaks around $\mathrm{E}_{\mathrm{F}}$. In the region of VBs, the increase of the DOS near the band edge for Zr-doped TiCoSb systems is much larger than that of TiCoSb compound throughout the energy region. These behaviors can make the effective mass of carriers increasing, and then improve the Seebeck coefficients of Zr-doped systems. Therefore, Zr-doping can improve the electronic transport properties of TiCoSb half-Heusler compounds.

Figure 6 shows the DOS of Hf-doped TiCoSb half-Heusler compounds near Fermi energy ( $\left.E_{F}\right)$ level. The calculated DOS of these doped compounds are normalized in this section. As can be seen from Figure 6(b), the DOS of Ti/HfCoSb half-Heusler compounds both exhibit sharp peaks around $\mathrm{E}_{\mathrm{F}}$. If one can dope these two compounds, we may be able to move $\mathrm{E}_{\mathrm{F}}$ to the region of high DOS. For the DOSs of $\mathrm{Ti}_{0.75} \mathrm{Hf}_{0.25} \mathrm{CoSb}$, $\mathrm{Ti}_{0.5} \mathrm{Hf}_{0.5} \mathrm{CoSb}$ and $\mathrm{Ti}_{0.25} \mathrm{Hf}_{0.75} \mathrm{CoSb}$ compounds, $\mathrm{E}_{\mathrm{F}}$ slightly move down to the valence band. This means that they may have the ability to contain more carriers than TiCoSb, and leading to electrical conductivity increase. Moreover, the amplitudes of the DOS in the region of VB for Hf-doped systems show a similar but slightly higher value than TiCoSb, which can increase the effective mass of carriers, and then improve the Seebeck coefficients of Hf-doped systems. Hence, Hf-doping can improve the electronic transport properties of TiCoSb half-Heusler compounds.

\subsection{Effect of M-Dopant on Phonon Transport Properties}

Based on the calculated band structures and density of states, we are able to evaluate the phonon dispersion relations based on the harmonic force constants obtained by the Phonopy package. This method has been successfully used in previous work [30]. To get reliable results, a $4 \times 4 \times 4$ supercell for Ti/Zr/HfCoSb and a $3 \times 3 \times 1$ supercell for $\mathrm{Ti}_{0.5} \mathrm{M}_{0.5} \mathrm{CoSb}$ are used in our calculations. The calculated phonon dispersion relations of undoped and doped TiCoSb compounds are presented in Figure 7. For Ti/Zr/HfCoSb, the three atoms in the primitive 


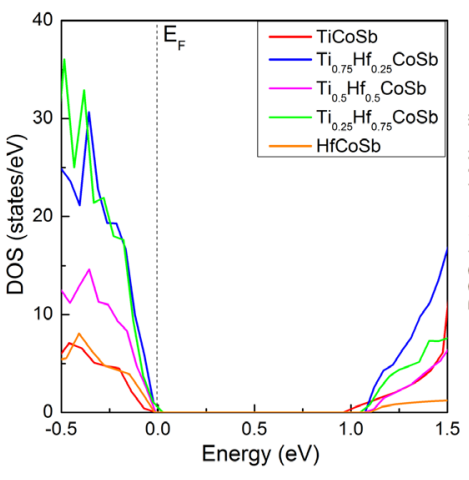

(a)

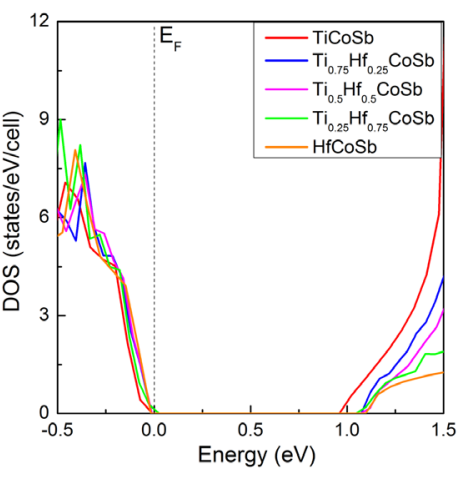

(b)

Figure 6. The density of states of undoped and Hf-doped TiCoSb without normalized (a) and with normalized (b).

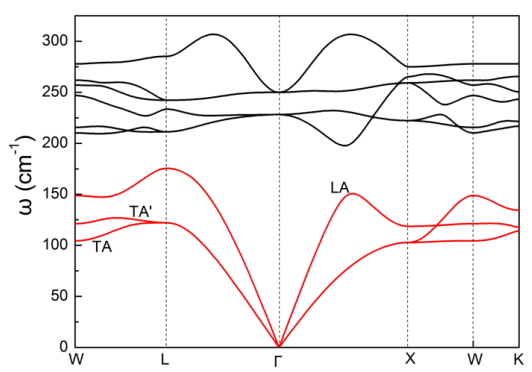

(a)

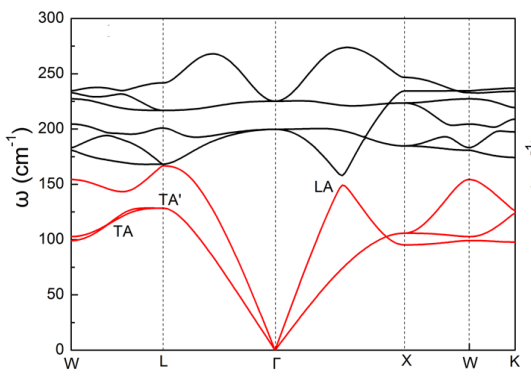

(b)

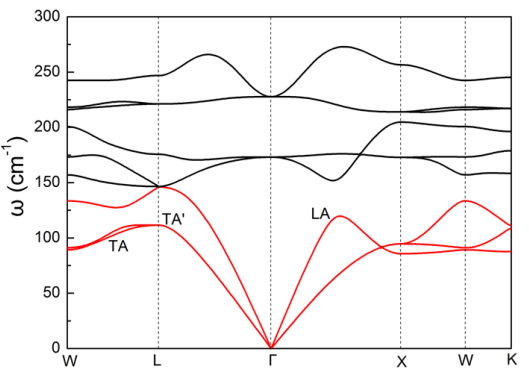

(c)

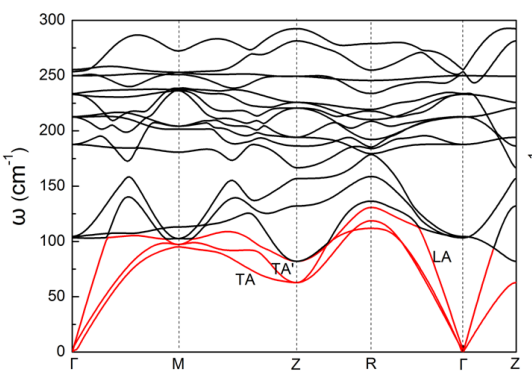

(d)

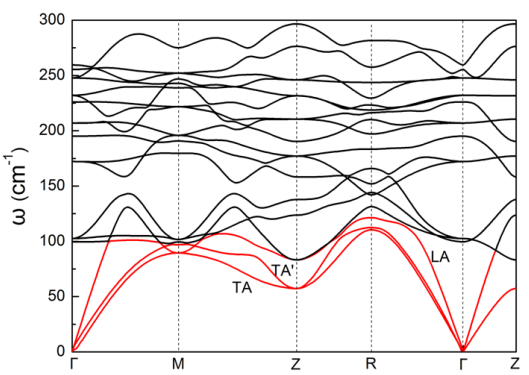

(e)

Figure 7. The phonon dispersion relations for: (a) TiCoSb; (b) ZrCoSb; (c) HfCoSb; (d) $\mathrm{Ti}_{0.5} \mathrm{Zr}_{0.5} \mathrm{CoSb}$; (e) $\mathrm{Ti}_{0.5} \mathrm{Hf}_{0.5} \mathrm{CoSb}$ along the high-symmetry lines calculated using the Phonopy package [34]. The acoustic modes are highlighted in red.

cell give rise to nine phonon branches, three acoustic modes (one longitudinal and two transverse branches) and six optical modes (two longitudinal and four transverse branches). Figures 7(a)-(c) show the phonon dispersion relations of $\mathrm{Ti} / \mathrm{Zr} / \mathrm{HfCoSb}$ compounds crystal along the high-symmetry lines. It can be seen that the acoustic branches of TiCoSb separate from optical branches with a small frequency gap. Unlike TiCoSb, the acoustic branches of $\mathrm{Zr} / \mathrm{HfCoSb}$ show mixing with the optical branch. In this section, $\mathrm{Ti}_{0.5} \mathrm{M}_{0.5} \mathrm{CoSb}$ will be investigated due to their relatively better electronic transport properties among the doped systems. Figure 7(e) and Figure 7 (d) show the phonon dispersion relations of $\mathrm{Ti}_{0.5} \mathrm{Hf}_{0.5} \mathrm{CoSb}$ and $\mathrm{Ti}_{0.5} \mathrm{Hf}_{0.5} \mathrm{CoSb}$ compounds, respectively. The six atoms in the primitive cell give rise to eighteen phonon branches, three acoustic modes and fifteen optical modes, where the acoustic branches overlap show significant mixing with optical branches. The larger mass of $\mathrm{Zr} / \mathrm{Hf}$ relative to $\mathrm{Ti}$ lowers the optical modes and induces mixing with the acoustic branches. And the frequencies of optical and acoustic modes are obviously decreased. The general features are consistent with phonon dispersion relations reported for other half-Heusler compounds such as ZrCoSb [31].

According to the description of lattice thermal conductivity $\left(\kappa_{l}=1 / 3 C_{v} v_{s} l\right)$ borrowed from the dynamical theory of glass molecules [32], it is anticipated that $\kappa_{l}$ is proportional to lattice heat capacity $\left(C_{v}\right)$, the phonon velocity $\left(v_{s}\right)$ and the mean free path of phonon $(l)$. Figure 8 reveals the relation of the calculated heat capacity 


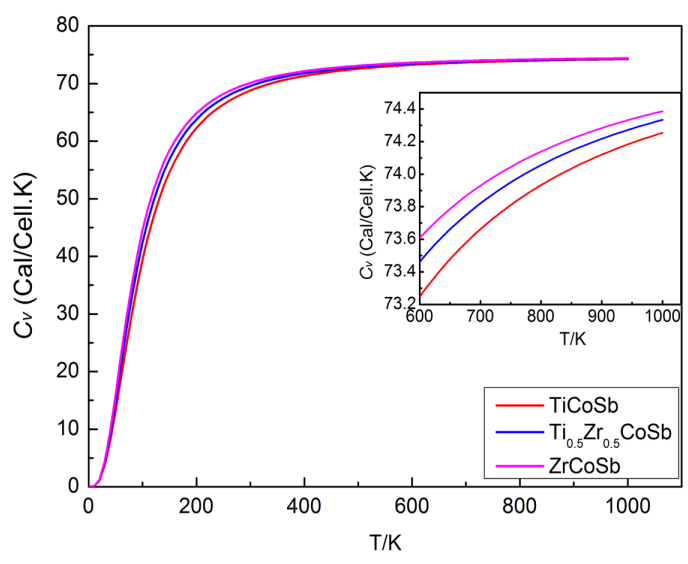

(a)

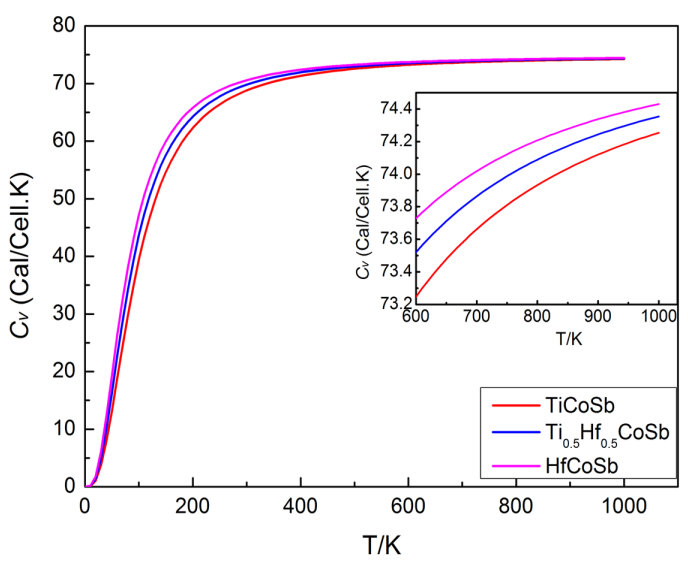

(b)

Figure 8. The relation of the calculated heat capacity and the temperature for: (a) Zr-doped and undoped TiCoSb; (b) Hfdoped and undoped TiCoSb.

and the temperature for M-doped TiCoSb systems. It can be seen that the lattice heat capacity increases rapidly with the temperature in the initial stage. The lattice heat capacity approaches a stable value when the temperature exceeds $400 \mathrm{~K}$. Besides, with the increasing of $\mathrm{M}$ doping concentration, the lattice heat capacities of doped systems have small increases. Although M-doping has slight effects on the lattice heat capacity of TiCoSb, the detailed understanding of the effect on the phonon velocity and the mean free path requires a further investigation. Moreover, the results of our calculations are in good agreement with the experimental results [25] [33] [34], and the current framework can be useful to characterize complicated crystal structures of thermoelectric materials. Finally, the present framework is beneficial to optimize the material composition or to design new phonon-engineered crystals, which will be the subject of our future work.

\section{Conclusion}

In summary, first-principles calculations were performed to study the $\mathrm{M}$-doping ( $\mathrm{M}=\mathrm{Zr}$, Hf) effects on the electronic structures and thermoelectric performance of TiCoSb under different M doping concentrations. With the increasing of $\mathrm{M}$ doping concentration, the calculated lattice constants of doped systems increase gradually. The formation energy is used to estimate the energetic stability of the M-doped systems. The results characterize that the formation enthalpies of these compounds are all negative, indicating that they can exist stably. The band structures analysis shows that substituting $\mathrm{Ti}$ with $\mathrm{Zr} / \mathrm{Hf}$ does not change the band structures of these systems significantly. Most of the M-doped systems have a lower band gap value than that of TiCoSb; especially the doped compound, $\mathrm{Ti}_{0.5} \mathrm{Zr}_{0.5} \mathrm{CoSb}$, has the lowest narrow gap of $0.971 \mathrm{eV}$. Besides, the amplitudes of the DOS in the region of the valence bands for M-doped systems show a similar but slightly higher value than TiCoSb. Those suggest that these compounds could have better thermoelectric performance than TiCoSb. The phonon dispersion relations show that the larger mass of $\mathrm{Zr} / \mathrm{Hf}$ relative to Ti lowers the optical modes and induces mixing with the acoustic branches. With the increasing of $\mathrm{M}$ doping concentration, the lattice heat capacities of doped systems are slightly increased. Finally, the present framework is beneficial to characterize complicated crystal structures of thermoelectric materials and optimize the material composition.

\section{Acknowledgements}

The financial support by the Natural Science Foundation of China (No. 11504228) and the Innovation Program of Shanghai Municipal Education Commission, China (10YZ172) and Subjects Construction Program of Shanghai University of Engineering Science, China (2012gp43) are gratefully appreciated.

\section{References}

[1] Zou, D.F., Xie, S.H., Liu, Y.Y., Lin, J.G. and Li, J.Y. (2013) Electronic Structure and Thermoelectric Properties of Half-Heusler $\mathrm{Zr}_{0.5} \mathrm{Hf}_{0.5} \mathrm{NiSn}$ by First-Principles Calculations. Journal of Applied Physics, 113, Article ID: 193705. http://dx.doi.org/10.1063/1.4804939 
[2] Ding, G.Q., Gao, G.Y. and Yao, K.L. (2014) Thermoelectric Performance of Half-Heusler Compounds MYSb (M = Ni, Pd, Pt). Journal of Physics D: Applied Physics, 47, Article ID: 385305.

http://dx.doi.org/10.1088/0022-3727/47/38/385305

[3] Geng, H.Y., Meng, X.F., Zhang, H. and Zhang, J. (2014) Lattice Thermal Conductivity of Nanograined Half-Heusler Solid Solutions. Applied Physics Letters, 104, Article ID: 202104. http://dx.doi.org/10.1063/1.4879248

[4] Culp, S.R., Poon, S.J., Hickman, N., Tritt, T.M. and Blumm, J. (2006) Effect of Substitutions on the Thermoelectric Figure of Merit of Half-Heusler Phases at $800^{\circ} \mathrm{C}$. Applied Physics Letters, 88, Article ID: 042106. http://dx.doi.org/10.1063/1.2168019

[5] Bhattacharya, S., Skove, M., Russell, M., Tritt, T., Xia, Y., Ponnambalam, V., Poon, S. and Thadhani, N. (2008) Effect of Boundary Scattering on the Thermal Conductivity of TiNiSn-Based Half-Heusler Alloys. Physical Review B, 77, Article ID: 184203. http://dx.doi.org/10.1103/physrevb.77.184203

[6] Ouardi, S., Fecher, G.H., Felser, C., Blum, C.G.F., Bombor, D., Hess, C., Wurmehl, S., Büchner, B. and Ikenaga, E. (2011) Transport and Thermal Properties of Single- and Polycrystalline $\mathrm{NiZr}_{0.5} \mathrm{Hf}_{0.5} \mathrm{Sn}$. Applied Physics Letters, 99, Article ID: 152112. http://dx.doi.org/10.1063/1.3651484

[7] Liu, C.R. and Li, J.B. (2011) Thermoelectric Properties of ZnO Nanowires: A First Principle Research. Physics Letters A, 375, 2878-2881. http://dx.doi.org/10.1016/j.physleta.2011.06.024

[8] Kimura, Y., Ueno, H. and Mishima, Y. (2009) Thermoelectric Properties of Directionally Solidified Half-Heusler $\left(\mathrm{M}_{0.5}{ }^{\mathrm{a}}, \mathrm{M}_{0.5}{ }^{\mathrm{b}}\right) \mathrm{NiSn}\left(\mathrm{M}^{\mathrm{a}}, \mathrm{M}^{\mathrm{b}}=\mathrm{Hf}, \mathrm{Zr}, \mathrm{Ti}\right)$ Alloys. Journal of Electronic Materials, 38, 934-939. http://dx.doi.org/10.1007/s11664-009-0710-X

[9] Xie, H.H., Wang, H., Pei, Y.Z., Fu, C.G., Liu, X.H., Snyder, G.J., Zhao, X.B. and Zhou, T.J. (2013) Beneficial Contribution of Alloy Disorder to Electron and Phonon Transport in Half-Heusler Thermoelectric Materials. Advanced Functional Materials, 23, 5123-5130. http://dx.doi.org/10.1002/adfm.201300663

[10] Uher, C., Yang, J., Hu, S., Morelli, D.T. and Meisner, G.P. (1999) Transport Properties of Pure and Doped MNiSn (M = Zr, Hf). Physical Review B, 59, 8615. http://dx.doi.org/10.1103/PhysRevB.59.8615

[11] Hohl, H., Ramirez, A.P., Goldmann, C., Ernst, G., Wolfing, B. and Bucher, E. (1999) Efficient Dopants for ZrNiSnBased Thermoelectric Materials. Journal of Physics: Condensed Matter, 11, 1697-1710. http://dx.doi.org/10.1088/0953-8984/11/7/004

[12] Xie, W.J., Jin, Q. and Tang, X.F. (2008) The Preparation and Thermoelectric Properties of $\mathrm{Ti}_{0.5} \mathrm{Zr}_{0.25} \mathrm{Hf}_{0.25} \mathrm{Co}_{1-x} \mathrm{Ni}_{\chi} \mathrm{Sb}$ Half-Heusler Compounds. Journal of Applied Physics, 103, Article ID: 043711. http://dx.doi.org/10.1063/1.2885113

[13] Huang, X.Y., Xu, Z. and Chen, L.D. (2004) The Thermoelectric Performance of $\mathrm{ZrNiSn} / \mathrm{ZrO}_{2}$ Composites. Solid State Communication, 130, 181-185. http://dx.doi.org/10.1016/j.ssc.2004.02.001

[14] Sakurada, S. and Shutoh, N. (2005) Effect of Ti Substitution on the Thermoelectric Properties of (Zr,Hf)NiSn HalfHeusler Compounds. Applied Physics Letters, 86, Article ID: 082105. http://dx.doi.org/10.1063/1.1868063

[15] Lindsay, L., Broido, D.A. and Reinecke, T.L. (2013) Ab initio Thermal Transport in Compound Semiconductors. Physical Review B, 87, Article ID: 165201. http://dx.doi.org/10.1103/PhysRevB.87.165201

[16] Nomura, M., Nakagawa, J., Kage, Y., Maire, J., Moser, D. and Paul, O. (2015) Thermal Phonon Transport in Silicon Nanowires and Two-Dimensional Phononic Crystal Nanostructures. Applied Physics Letters, 106, Article ID: 143102. http://dx.doi.org/10.1063/1.4917036

[17] Yang, J., Meisner, G.P. and Chen, L.D. (2004) Strain Field Fluctuation Effects on Lattice Thermal Conductivity of ZrNiSn-Based Thermoelectric Compounds. Applied Physics Letters, 85, 1140. http://dx.doi.org/10.1063/1.1783022

[18] Tian, Z.T., Garg, J., Esfarjani, K., Shiga, T., Shiomi, J. and Chen, G. (2012) Phonon Conduction in PbSe, PbTe, and $\mathrm{PbTe}_{1-\chi} \mathrm{Se}_{\mathrm{x}}$ from First-Principles Calculations. Physical Review B, 85, Article ID: 184303. http://dx.doi.org/10.1103/PhysRevB.85.184303

[19] Sekimoto, T., Kurosaki, K., Muta, H. and Yamanaka, S. (2006) Thermoelectric and Thermophysical Properties of $\operatorname{ErPdX}(\mathrm{X}=\mathrm{Sb}$ and Bi) Half-Heusler Compounds. Journal of Applied Physics, 99, Article ID: 103701. http://dx.doi.org/10.1063/1.2196109

[20] Kresse, G. and Furthmüller, J. (1996) Efficiency of Ab-Initio Total Energy Calculations for Metals and Semiconductors Using a Plane-Wave Basis Set. Computer Materials Science, 6, 15-50. http://dx.doi.org/10.1016/0927-0256(96)00008-0

[21] Kresse, G. and Furthmüller, J. (1996) Efficient Iterative Schemes for Ab Initio Total-Energy Calculations Using a Plane-Wave Basis Set. Physical Review B, 54, Article ID: 011169. http://dx.doi.org/10.1103/PhysRevB.54.11169

[22] Kresse, G. and Hafner, J. (1993) Ab initio Molecular Dynamics for Liquid Metals. Physical Review B, 47, $558-561$. http://dx.doi.org/10.1103/PhysRevB.47.558

[23] Perdew, J.P., Burke, K. and Ernzerhof, M. (1996) Generalized Gradient Approximation Made Simple. Physical Review 
Letter, 77, 3865-3868. http://dx.doi.org/10.1103/PhysRevLett.77.3865

[24] Wang, L.L., Miao, L., Wang, Z.Y., Wei, W., Xiong, R., Liu, H.J., Shi, J. and Tang, X.F. (2009) Thermoelectric Performance of Half-Heusler Compounds TiNiSn and TiCoSb. Journal of Applied Physics, 105, Article ID: 013709. http://dx.doi.org/10.1063/1.3056384

[25] Sekimoto, T., Kurosaki, K., Muta, H. and Yamanaka, S. (2005) Thermoelectric Properties of (Ti,Zr,Hf)CoSb Type Half-Heusler Compounds. Materials Transactions, 46, 1481-1484. http://dx.doi.org/10.2320/matertrans.46.1481

[26] Yang, J., Li, H.M., Wu, T., Zhang, W.Q., Chen, L.D. and Yang, J.H. (2008) Evaluation of Half-Heusler Compounds as Thermoelectric Materials Based on the Calculated Electrical Transport Properties. Advanced Functional Materials, 18, 2880-2888. http://dx.doi.org/10.1002/adfm.200701369

[27] Khein, A., Singh, D.J. and Umrigar, C.J. (1995) All-Electron Study of Gradient Corrections to the Local-Density Functional in Metallic Systems. Physical Review B, 51, 4105-4109. http://dx.doi.org/10.1103/PhysRevB.51.4105

[28] Song, Y. and Guo, A.X. (2006) Electronic Structure, Stability and Bonding of the Li-N-H Hydrogen Storage System. Physical Review B, 74, Article ID: 195120. http://dx.doi.org/10.1103/PhysRevB.74.195120

[29] Lee, M.S., Poudeu, F.P. and Mahanti, S.D. (2011) Electronic Structure and Thermoelectric Properties of Sb-Based Semiconducting Half-Heusler Compounds. Physical Review B, 83, Article ID: 085204. http://dx.doi.org/10.1103/PhysRevB.83.085204

[30] Ding, G.Q., Gao, G.Y. and Yao, K.L. (2015) Examining the Thermal Conductivity of the Half-Heusler Alloy TiNiSn by First-Principles Calculations. Journal of Physics D: Applied Physics, 48, Article ID: 235302. http://dx.doi.org/10.1088/0022-3727/48/23/235302

[31] Shiomi, J., Esfarjani, K. and Chen, G. (2011) Thermal Conductivity of Half-Heusler Compounds from First-Principles Calculations. Physical Review B, 84, Article ID: 104302. http://dx.doi.org/10.1103/PhysRevB.84.104302

[32] Togo, A., Oba, F. and Tanaka, I. (2008) First-Principles Calculations of the Ferroelastic Transition between RutileType and $\mathrm{CaCl}_{2}$-Type $\mathrm{SiO}_{2}$ at High Pressures. Physical Review B, 78, Article ID: 134106 . http://dx.doi.org/10.1103/PhysRevB.78.134106

[33] Xia, Y., Bhattacharya, S., Ponnambalam, V., Pope, A.L., Poon, S.J. and Tritt, T.M. (2000) Thermoelectric Properties of Semimetallic (Zr, Hf)CoSb Half-Heusler Phases. Journal of Applied Physics, 88, 1952. http://dx.doi.org/10.1063/1.1305829

[34] Qiu, P.F., Huang, X.Y., Chen, X.H. and Chen, L.D. (2009) Enhanced Thermoelectric Performance by the Combination of Alloying and Doping in TiCoSb-Based Half-Heusler Compounds. Journal of Applied Physics, 106, Article ID: 103703. http://dx.doi.org/10.1063/1.3238363 